\title{
Hypoperfusion of an Entire Cerebral Hemisphere - Stroke or Postictal Deficit?
}

\author{
Filip Scheperjans $^{\mathrm{a}}$ Heli Silvennoinen ${ }^{\mathrm{b}}$ Satu Mustanoja \\ Maarit Palomäki ${ }^{b} \quad$ Nina Forss ${ }^{a}$ \\ ${ }^{a}$ Department of Neurology, Helsinki University Central Hospital, and bepartment \\ of Radiology, University of Helsinki and HUS Radiology (Medical Imaging Center), \\ Helsinki, Finland
}

\section{Key Words}

$\mathrm{CT} \cdot \mathrm{CT}$ angiography $\cdot$ Malignant stroke $\cdot$ Neuroradiology $\cdot$ Perfusion imaging $\cdot$ Postictal state $\cdot$ Thrombolysis

\begin{abstract}
The clinical differential diagnosis between ischemic stroke and postictal deficit is sometimes challenging. If the clinical presentation is inconclusive, perfusion imaging can help to identify stroke patients for thrombolysis therapy. However, also epileptic phenomena may alter cerebral perfusion. Hypoperfusion spreading beyond the borders of cerebrovascular territories is usually considered suggestive of an etiology other than stroke. We present a patient whose clinical symptoms suggested a postictal deficit rather than an acute stroke. CT perfusion imaging showed hypoperfusion of the entire left cerebral hemisphere covering all vascular territories. CT angiography revealed occlusions in the ipsilateral internal carotid artery and in the circle of Willis as the cause of the global hypoperfusion. The patient was treated with i.v. thrombolysis and recovered with moderate disability. This is the first description of hyperacute ischemia of an entire cerebral hemisphere and its treatment with thrombolysis. It demonstrates the potential of modern neuroimaging in identifying atypically presenting strokes and shows that i.v. thrombolysis can be effectively and safely used to treat such potentially fatal insults.
\end{abstract}

\section{Introduction}

The clinical differential diagnosis between ischemic stroke and postictal deficit is sometimes challenging. On the one hand, about $5 \%$ of patients treated with thrombolysis have actually had an epileptic seizure mimicking stroke [1], on the other hand, $2-7 \%$ of strokes are accompanied by an epileptic seizure at onset $[2,3]$. If the clinical presentation 
is inconclusive, perfusion imaging can help to differentiate between these two conditions. However, also epileptic phenomena can be associated with altered cerebral perfusion [4]. We present a case of complete hemispheric ischemia that could have easily been mistaken for a postictal deficit based on the clinical findings alone, with potentially fatal consequences.

\section{Case Report}

Our patient is a 61-year-old man with a history of untreated hypertension, prediabetes, smoking, and alcohol abuse. Ambulance staff reported that after a fall his level of consciousness was decreased, grip force was bilaterally reduced and the right leg was weak. On arrival, he was somnolent, aphasic and turned his head and gaze intermittently to the right. Spontaneous movements were observed in all extremities. There was no facial asymmetry. He extended the arms and flexed the legs symmetrically to pain. Right plantar reflex was positive. Some myoclonic jerks were seen in the extremities. His Glasgow Coma Scale level was 8 and the National Institutes of Health Stroke Scale (NIHSS) score was 22.

Noncontrast CT (Siemens Somatom Definition AS+ 128 slice) showed no acute pathology but there were old infarcts in the frontal and occipital watershed regions of the left hemisphere and bilaterally in the corona radiata (fig. 1). In contrast, CT perfusion imaging revealed massive hypoperfusion of the whole left hemisphere encompassing the territories of the anterior, middle, and posterior cerebral arteries (fig. 1). CT angiography (CTA) showed atherosclerotic occlusion of the left proximal internal carotid artery (ICA; fig. 2A). Intracranially, there was an occlusion at the bifurcation of the left posterior communicating artery ( $\mathrm{PCoA})$ and posterior cerebral artery (PCA), with some retrograde filling distally (fig. 2B). There were no signs of vertebrobasilar occlusion.

The decreased cerebral blood flow and prolonged mean transit time suggested acute ischemia of the entire left hemisphere, while cerebral blood volume (CBV) reductions were visible only in the watershed regions. This mismatch was suggestive of a large penumbra (fig. 1). Therefore, the patient was treated with i.v. thrombolysis at $2.5 \mathrm{~h}$ after symptom onset although clinical symptoms were atypical for hemispheric ischemia.

At $18 \mathrm{~h}$ after treatment, the NIHSS score had decreased to 14 . Control CT revealed acute infarcts in the left PCA territory and around the old frontal watershed infarct (fig. 3). EEG showed no epileptiform activity. Control CTA at $48 \mathrm{~h}$ showed recanalization of the left PCoA and PCA, while the left ICA was still occluded (fig. 2B). No source of cardiogenic embolization was found during further workup. At 3 months, the patient's language skills were close to normal, he had right-sided hemianopia and neglect without major hemiparesis (modified Rankin Scale level 3).

\section{Discussion}

Simultaneous ischemia in ipsilateral anterior and posterior cerebrovascular territories occurs in about $1.5-3 \%$ of strokes $[5,6]$, but we found only one reported case in the literature with involvement of an entire cerebral hemisphere [7]. That patient had a complete infarction with typical symptoms of malignant stroke and died from cerebral herniation shortly after imaging. The stroke was caused by a persistent fetal PCA supplied exclusively from the ipsilateral ICA that became acutely occluded.

Why did our patient not have typical symptoms of major hemispheric stroke such as gaze deviation, facial palsy, and total hemiplegia? While we cannot give a definite answer to this question, we propose that the discrepancy between the imaging findings and the clinical symptoms could be explained by a chronic occlusion of the left ICA (fig. 2A). In cases of slowly developing ICA occlusion, blood is supplied to the anterior circulation primarily through collateral vessels of the circle of Willis [8]. In our patient, the anterior 
communicating artery was poorly developed. If the patient's ICA was chronically occluded, the left anterior circulation would be dependent entirely on blood supply from the vertebrobasilar vessels through the prominent PCoA, likely leading to chronic hypoperfusion [8,9]. In rats, chronic cerebral hypoperfusion induces compensatory changes in brain tissue such as vascular remodeling and metabolic adaptation [10, 11]. Therefore, in previously hypoperfused brains, tissue damage after acute ischemia is less severe and is reflected by milder motor disability compared to healthy brains [10]. It may be that in our patient, a chronic hypoperfusion of the left hemisphere had induced vascular remodeling and an increase of the vascular reserve possibly involving leptomeningeal collaterals $[8,10]$. Furthermore, the nervous tissue may have been to some degree metabolically adapted increasing its tolerance to acute ischemia $[11,12]$. It seems possible that such factors may have contributed to the patient's atypically mild focal signs when the hypoperfusion was acutely aggravated by the PCoA occlusion $[8,10-$ 12]. While this explanation is somewhat speculative, there are two important points supporting our hypothesis of chronic ICA occlusion. First, old watershed infarcts were present suggesting preceding hemodynamically relevant ICA pathology (fig. 1). Second, while the ICA remained occluded, recanalization of only the PCoA was sufficient to prevent major infarction in the anterior circulation (fig. 3). This would be very unlikely in a case of acute tandem occlusion.

Absence of a typical hemispheric stroke syndrome combined with a profoundly decreased level of consciousness and myoclonia raised the possibility of epileptic phenomena underlying the patient's symptoms. While ictal activity is considered to elicit hyperperfusion $[13,14]$, contradicting results have been published regarding postictal perfusion changes $[15,16]$ possibly due to the temporal dynamics of postictal brain perfusion with phases of hypo- and hyperperfusion [17]. Seizure-related cerebral perfusion changes are typically not confined to a cerebrovascular territory. Hypoperfusion of an entire cerebral hemisphere has been reported in few cases with postictal Todd's paresis $[4,18,19]$, but never before in stroke. In our case, the spatial distribution pattern of hypoperfusion was therefore misleading. However, postictally $\mathrm{CBV}$ is usually decreased, whereas in our patient CBV was normal outside of the watershed regions (fig. 1) [4]. This constellation together with the vessel occlusion in the circle of Willis shown by CTA confirmed the diagnosis of acute stroke (fig. 2B) [3].

Our case demonstrates that acute ischemia of a whole cerebral hemisphere is possible without vascular anatomical variants. With preexisting severe atherosclerotic disease of the cerebral vasculature, the clinical symptoms are not necessarily the simple addition of anterior, middle, and posterior cerebral artery syndromes and can easily be misinterpreted. In cases of complete hemispheric hypoperfusion, stroke should be considered as a possible etiology and additional CTA should be performed. If a relevant penumbra and vessel occlusion can be identified [3], rapid revascularization treatment may save patients from a fatal outcome. 


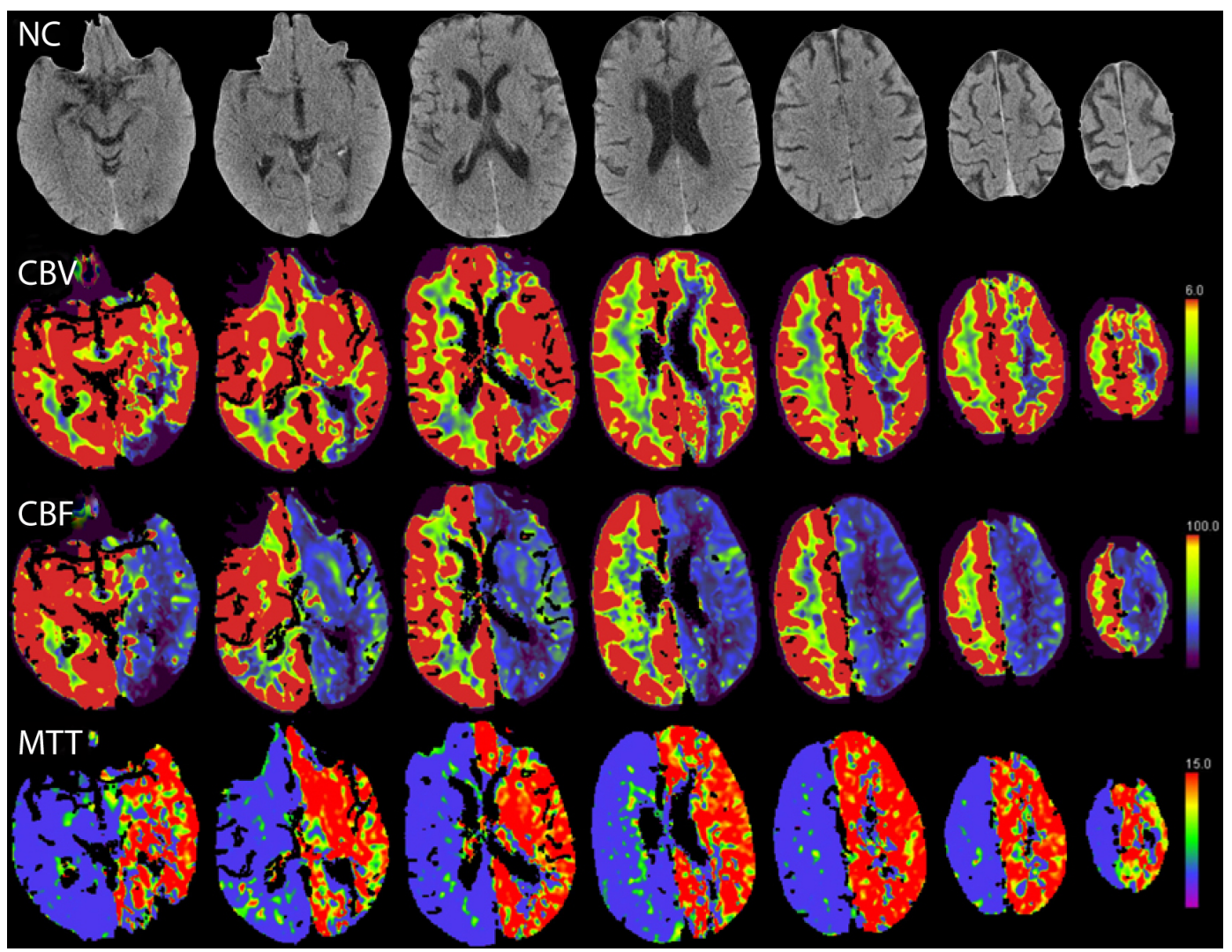

Fig. 1. The upper row shows the primary noncontrast (NC) CT revealing old infarcts in the frontal and occipital watershed regions on the left and bilaterally in the corona radiata. Below are CT perfusion maps showing pronounced hypoperfusion of the entire left cerebral hemisphere as decreased cerebral blood flow (CBF) and prolonged mean transit time (MTT). However, CBV is decreased only in the watershed regions suggesting that most of the ischemic area is salvageable tissue (penumbra). 

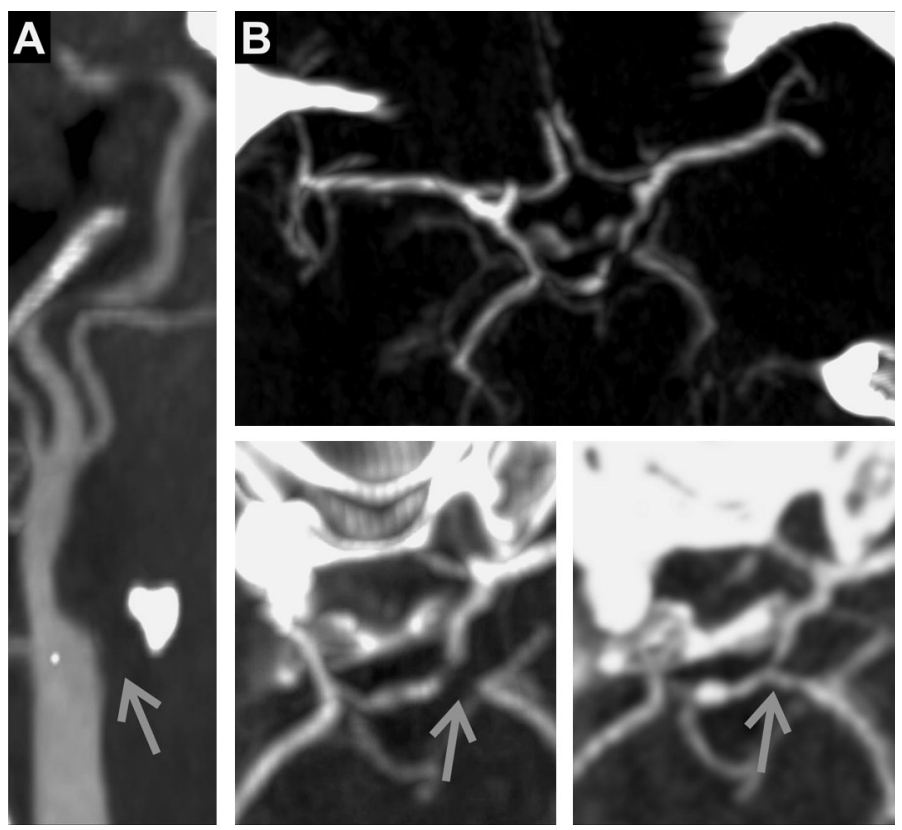

Fig. 2. A CTA of the left common carotid artery and its branches. Filling occurs only in the external carotid artery, whereas the ICA is occluded (arrow). The large calcified plaque suggests atherosclerotic pathology. B CTA of the circle of Willis. The anterior communicating artery is poorly developed, whereas the PCoAs are prominent. No occlusion is seen in the left middle cerebral artery. The insets below show a magnification of the findings before (left) and 2 days after thrombolysis (right). Arrows point to the occlusion at the bifurcation of the left PCoA and the PCA that was recanalized.

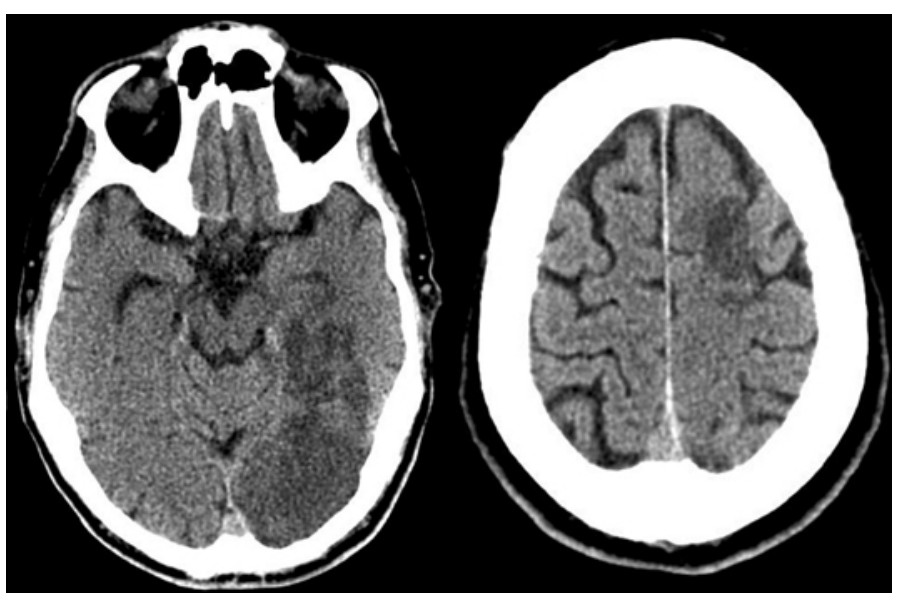

Fig. 3. Noncontrast CT scan $18 \mathrm{~h}$ after thrombolysis showing enlargement of the previous frontal watershed infarct and a new infarct in the PCA territory. 


\section{References}

1 Chernyshev OY, Martin-Schild S, Albright KC, Barreto A, Misra V, Acosta I, Grotta JC, Savitz SI: Safety of tPA in stroke mimics and neuroimaging-negative cerebral ischemia. Neurology 2010;74:1340-1345.

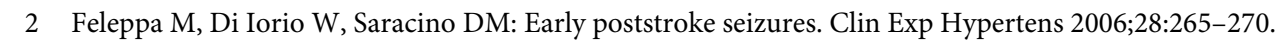

-3 Sylaja PN, Dzialowski I, Krol A, Roy J, Federico P, Demchuk AM: Role of CT angiography in thrombolysis decision-making for patients with presumed seizure at stroke onset. Stroke 2006;37:915-917.

-4 Gelfand JM, Wintermark M, Josephson SA: Cerebral perfusion-CT patterns following seizure. Eur J Neurol 2010;17:594-601.

5 Roh JK, Kang DW, Lee SH, Yoon BW, Chang KH: Significance of acute multiple brain infarction on diffusionweighted imaging. Stroke 2000;31:688-694.

6 Yang JH, Choi HY, Nam HS, Kim SH, Han SW, Heo JH: Mechanism of infarction involving ipsilateral carotid and posterior cerebral artery territories. Cerebrovasc Dis 2007;24:445-451.

7 Kumar KR, Kleinig T: Neurological picture. 'Malignant' ischaemic stroke of an entire cerebral hemisphere. J Neurol Neurosurg Psychiatry 2009;80:87.

8 Liebeskind DS: Collateral circulation. Stroke 2003;34:2279-2284.

-9 Chaves CJ, Staroselskaya I, Linfante I, Llinas R, Caplan LR, Warach S: Patterns of perfusion-weighted imaging in patients with carotid artery occlusive disease. Arch Neurol 2003;60:237-242.

10 Kim SH, Kim EH, Lee BI, Heo JH: Chronic cerebral hypoperfusion protects against acute focal ischemia, improves motor function, and results in vascular remodeling. Curr Neurovasc Res 2008;5:28-36.

-11 Mracskó É, Hugyecz M, Institoris A, Farkas E, Bari F: Changes in pro-oxidant and antioxidant enzyme levels during cerebral hypoperfusion in rats. Brain Res 2010;1321:13-19.

12 Durukan A, Tatlisumak T: Preconditioning-induced ischemic tolerance: a window into endogenous gearing for cerebroprotection. Exp Transl Stroke Med 2010;2:2.

-13 Hauf M, Slotboom J, Nirkko A, von BF, Ozdoba C, Wiest R: Cortical regional hyperperfusion in nonconvulsive status epilepticus measured by dynamic brain perfusion CT. AJNR Am J Neuroradiol 2009;30:693-698

14 Masterson K, Vargas MI, Delavelle J: Postictal deficit mimicking stroke: role of perfusion CT. J Neuroradiol 2009;36:48-51.

-15 Kimura M, Sejima H, Ozasa H, Yamaguchi S: Technetium-99m-HMPAO SPECT in patients with hemiconvulsions followed by Todd's paralysis. Pediatr Radiol 1998;28:92-94.

-16 Wiest R, von Bredow F, Schindler K, Schauble B, Slotboom J, Brekenfeld C, Remonda L, Schroth G, Ozdoba C: Detection of regional blood perfusion changes in epileptic seizures with dynamic brain perfusion CT - a pilot study. Epilepsy Res 2006;72:102-110.

17 Leonhardt G, de Greiff A, Weber J, Ludwig T, Wiedemayer H, Forsting M, Hufnagel A: Brain perfusion following single seizures. Epilepsia 2005;46:1943-1949.

18 Mathews MS, Smith WS, Wintermark M, Dillon WP, Binder DK: Local cortical hypoperfusion imaged with CT perfusion during postictal Todd's paresis. Neuroradiology 2008;50:397-401.

19 Rupprecht S, Schwab M, Fitzek C, Witte OW, Terborg C, Hagemann G: Hemispheric hypoperfusion in postictal paresis mimics early brain ischemia. Epilepsy Res 2010;89:355-359. 